\title{
Congenital Syphilis After Treatment of Maternal Syphilis With a Penicillin Regimen Exceeding CDC Guidelines
}

\author{
Craig S. Conover, ${ }^{1 *}$ Charles A. Rend, ${ }^{2}$ Grayson B. Miller, Jr., ${ }^{3}$ and \\ George P. Schmid ${ }^{4}$ \\ ${ }^{1}$ Illinois Department of Public Health, Division of Infectious Diseases, Chicago, IL \\ ${ }^{2}$ Virginia Department of Health, Hampton Health District, Hampton, VA \\ ${ }^{3}$ Virginia Department of Health, Office of Epidemiology, Richmond, VA \\ ${ }^{4}$ Division of STD/HIV Prevention, NCHSTP, Centers for Disease Control and Prevention, \\ Atlanta, GA
}

\begin{abstract}
Background: Although congenital syphilis usually occurs as a result of a failure to detect and treat syphilis in pregnant women, failures of the currently recommended regimen to prevent congenital syphilis have been reported.

Case: This report describes an infant with congenital syphilis despite maternal treatment with a regimen exceeding current CDC guidelines.

Conclusion: Regardless of the regimen used to treat syphilis during pregnancy, clinicians should recognize the possibility of occasional treatment failures and the importance of adequate follow-up of infants at risk for congenital syphilis. Infect. Dis. Obstet. Gynecol. 6:134-137, 1998.
\end{abstract}

(๑) 1998 Wiley-Liss, Inc.

congenital syphilis; maternal syphilis; syphilis in pregnancy

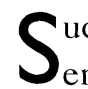
uccessful therapy of syphilis during pregnancy entails maternal cure as well as prevention or cure of congenital infection. Most cases of congenital syphilis occur as a result of failure to detect and treat syphilis in pregnant women, but failures of the recommended treatment regimen to prevent congenital syphilis have been reported. ${ }^{1-3} \mathrm{Al}-$ though some of these case reports probably represent congenital syphilis occurring as a result of undetected maternal reinfection after treatment, clinicians should recognize that penicillin regimens used to treat syphilis are not $100 \%$ curative. ${ }^{4} \mathrm{We}$ describe a case of congenital syphilis that occurred despite maternal treatment with a penicillin regimen exceeding current Centers for Disease Control and Prevention (CDC) treatment guidelines, highlighting the importance of adequate follow-up after birth for infants at risk for congenital syphilis.

\section{CASE REPORT}

A 35-year-old G2 P1 woman first attended a prenatal care clinic at 12 weeks gestational age. Routine syphilis screening was performed at this visit, and the RPR titer was 1:256 with a positive confirmatory MHA-TP. HIV test results were negative. She had no symptoms or signs of neurosyphilis.

The former affiliation for Craig S. Conover was Epidemic Intelligence Service, Division of Field Epidemiology, Centers for Disease Control, Atlanta, GA 30333.

*Correspondence to: Dr. Craig S. Conover, Department of Public Health, Division of Infectious Diseases, 160 N. Lasalle Street, Chicago, Illinois 60601. E-mail: cconover@idph.state.il.us 
The patient recalled that 2 months earlier she had developed a rash on her trunk and on her extremities which extended onto her palms. Two of her 3 sex partners during the previous year were located and evaluated for syphilis; both had negative RPR titers.

The patient stated she was sexually active with one partner throughout her pregnancy; he had also been evaluated during the original contact investigation and remained RPR-negative when retested. She was treated with benzathine penicillin $G, 2.4$ million units intramuscularly once weekly at 14,15 , and 16 weeks of gestation. The mother received no additional antibiotics during pregnancy. RPR titers (performed in the same laboratory) at 20, 28, and 36 weeks of gestation declined to $1: 128,1: 64$, and 1: 32 , respectively. At 41 weeks of gestation, an asymptomatic, female infant weighing 6.5 pounds was delivered without complications. At delivery, the mother's RPR titer remained at 1:32. The RPR titer on a heel-stick drawn from the infant at birth was weakly positive, and was reported by the laboratory on the day of discharge from the hospital. Information regarding the mother's history of syphilis during pregnancy was not available during a routine clinic appointment 6 weeks after birth, and the infant's RPR titer was not rechecked. At age 10 weeks, the infant developed a fever, rhinorrhea, and a diffuse copper-colored maculopapular rash. Congenital syphilis was not considered as a possibility, and the infant was diagnosed with a "viral syndrome." Two days later, a CBC was obtained during re-evaluation of the infant at another physician's office; the white blood cell count was $72,000 \mathrm{~mm}^{3}$, hematocrit $22.3 \%$, and platelet count $36,600 / \mathrm{mm}^{3}$. The infant was admitted to the hospital for further evaluation. On presentation, the infant had a fever of $101^{\circ}$ Farenheit, hepatomegaly, and a normal ophthalmologic exam. There was no evidence of sexual or other child abuse. Results of additional laboratory studies included an RPR titer of $>1: 256$, negative ELISA for HIV, and normal CSF profile with negative CSF VDRL. Radiographs of the extremities demonstrated metaphysitis and periosteal new bone formation, with a bony sequestrum in the right humerus. The infant was treated for congenital syphilis with 14 days of intravenous penicillin $(250,000$ units intravenously every 8 hours), and she recovered without further complications. At a follow-up visit to the STD clinic 8 weeks after the infant's hospitalization, the mother's RPR titer was 1:16, and HIV serology was again negative. At a subsequent follow-up visit to the STD clinic 6 months later, the mother's RPR titer had declined to $1: 4$.

\section{DISCUSSION}

Based on the history of rash 2 months prior to serodiagnosis, the infant's mother had early latent syphilis at the time she began treatment at the beginning of the second trimester of pregnancy. Clinical and laboratory parameters did not suggest reinfection with Treponema pallidum later in pregnancy, which might have resulted in the apparent fetal treatment failure. 'The decline in the mother's titers after delivery cannot be attributed to additional antibiotic treatment beyond that received in the prenatal clinic, as no other antibiotics were administered during pregnancy or the post-partum period. Also, the mother did not have HIV infection, which published case reports suggest might be associated with higher rates of syphilis treatment failure in non-pregnant patients. ${ }^{5}$ Despite treatment of the mother with three weekly doses of 2.4 million units of penicillin, the infant was born with congenital syphilis that was not diagnosed until 10 weeks after birth. Most syphilitic infants lack clinical manifestations at birth but nearly all cases are clinically manifest by 12 weeks. ${ }^{6}$ Factors contributing to the delayed diagnosis include unavailability of the infant's RPR results at birth to pediatricians providing subsequent outpatient care for the patient, and failure of several physicians to consider the diagnosis of congenital syphilis despite a "classic" presentation.

Current CDC treatment guidelines for treatment of syphilis in pregnancy specify using a regimen "appropriate for the stage of syphilis". 7 The usual treatment for early latent syphilis in adults is intramuscular benzathine penicillin G, 2.4 milliunits in a single dose. Recently, several investigators have questioned whether current treatment regimens for syphilis in pregnancy are adequate for preventing and curing fetal infection. ${ }^{2,8}$ Acknowledging current evidence is not sufficient to ascertain if the single dose regimen is optimal for preventing and treating fetal infections, the CDC's 1998 STD Treatment Guidelines note that some experts recommend additional therapy (e.g., a second dose of 2.4 million units of penicillin) 1 week 
after the initial dose, particularly for women in the third trimester of pregnancy and for women who have secondary syphilis during pregnancy. ${ }^{7}$

The effectiveness of any treatment regimen is likely to be dependent on a number of variables, such as stage of maternal syphilis, the severity of fetal infection, timing of treatment, and fetal tissue penicillin levels. ${ }^{4}$ Theoretically, severe fetal infection is most likely to occur with delayed treatment and during secondary maternal syphilis, when maternal spirochetemia is highest. More severe fetal infections are likely to cause more impairment of placental function, which might result in a reduction in fetal penicillin levels. The stage of pregnancy might also affect the likelihood of cure (e.g., fetal penicillin levels may be lower during the last trimester of pregnancy when maternal pharmacokinetics are altered by increased renal blood flow and penicillin's larger volume of distribution). ${ }^{4}$

Because penicillin therapy for maternal syphilis cannot be assumed to prevent or cure all cases of congenital syphilis, infants whose mothers were treated for syphilis during pregnancy should be evaluated clinically and serologically for evidence of congenital syphilis. In addition, pathologic exam of the placenta or umbilical cord is suggested if the infant's mother has reactive syphilis serology. Determining whether an asymptomatic infant has congenital syphilis at birth is often difficult, since (a) a serologic response may not be present at birth in some infants infected late in pregnancy because of insufficient time for an antibody response to develop, and (b) for infants with a positive syphilis serology, routine laboratory studies cannot distinguish the passively transferred maternal antibody from the fetal antibody. Although a neonatal titer that is $\geq 4$ times the maternal titer is highly suggestive of congenital syphilis, infected neonates may have titers lower than maternal titers. ${ }^{9}$

Given the limitations of conventional tests, improved methods for diagnosing congenital syphilis are desirable. Several ELISA-based tests have been developed that are designed to detect infant IgM antibody to Treponema pallidum but are not yet available for routine clinical laboratory use. ${ }^{9}$ PCRbased techniques for diagnosing congenital syphilis, first described in 1991, have excellent specificity but limited sensitivity for detection of positive specimens. ${ }^{10}$ No PCR-based test for the diagnosis of syphilis is commercially available at present.
A seroreactive infant, or an infant whose mother was seroreactive at delivery, who is not treated for congenital syphilis during the perinatal period, must receive careful follow-up examinations at 1, 2, 3,6 , and 12 months. Nontreponemal antibody titers should decline by 3 months of age and should be nonreactive by 6 months of age if the infant was not infected and the positive titers were the result of a passive transfer of antibody from the mother. If follow-up titers are stable or increasing, the infant should be re-evaluated, including a lumbar puncture, and fully treated. If treponemal antibody titers are positive at 12 months, full evaluation and treatment of the infant are recommended. Even if previous tests have been negative, a repeat serologic test should be performed in infants who develop symptoms, signs, or laboratory abnormalities consistent with congenital syphilis. ${ }^{7}$

Although well-documented fetal treatment failures are rare, data derived from clinical trials supporting current recommendations for treatment of syphilis in pregnancy are limited. It is unlikely that investigators will conduct trials large enough to detect a difference in the efficacy of current and alternative regimens for treatment of syphilis in pregnant women. Instead, assessment of the need for recommendations for alternative treatment is likely to be based primarily on evidence from large case-series documenting results with currently recommended therapy and studies of the pharmacokinetics of benzathine penicillin $G$ at different stages of pregnancy. Regardless of the regimen used to treat syphilis during pregnancy, clinicians should recognize the possibility of occasional treatment failures. Timely detection of these cases requires careful evaluation and follow-up of infants whose mothers were treated for syphilis during pregnancy.

\section{ACKNOWLEDGMENTS}

The authors thank Casey Riley, Gwen Hudgins, Wayne Lloyd, and Michael Wansky, D.V.M., for assistance with this investigation.

\section{REFERENCES}

1. Mascola L, Pelosi R, Alexander CE: Inadequate treatment of syphilis in pregnancy. Am J Obstet Gynecol 150:945-947, 1984.

2. Rawstrom SA, Bromberg K: Failure of recommended maternal therapy to prevent congenital syphilis. Sex Trans Dis 18:102-106, 1991 
3. Hardy JB, Hardy PH, Oppenheimer EH, Ryan SJ, Sheff $\mathrm{RN}$ : Failure of penicillin in a newborn with congenital syphilis. JAMA 212:1345-1349, 1970.

4. Rolfs RT: Treatment of syphilis, 1993. Clin Infect Dis 20:S23-S38, 1995.

5. Musher DM, Hammil RJ, Baughn RE: Effect of human immunodeficiency virus (HIV) infection on the course of syphilis and on the response to treatment. Ann Intern Med 113:872-881, 1990.

6. Schulz KF, Murphy FK, Patamuscon PP, Meheus AZ. Congenital syphilis, In: Holmes KK, et al. (eds): Sexually Transmitted Diseases. New York: McGraw Hill, pp 821-842, 1990.
7. CDC: Sexually transmitted diseases treatment guidelines. MMWR 47(RR-1):40-41, 1998.

8. Monif GR: Is current therapy for maternal syphilis inadequate for established fetal infection? Am J Obstet Gynecol 170:705, 1994.

9. Larsen S, Steiner B, Rudolph A: Laboratory diagnosis and interpretation of tests for syphilis. Clin Microbiol Rev 8:1-21, 1994.

10. Grimprel E, Sanchez G, Wendel J, et al.: Use of polymerase chain reaction and rabbit infectivity testing to detect Treponema pallidum in amniotic fluid. J Clin Microbiol 29:1711-1718, 1991. 


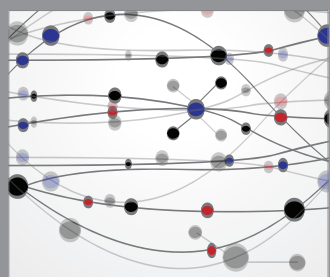

The Scientific World Journal
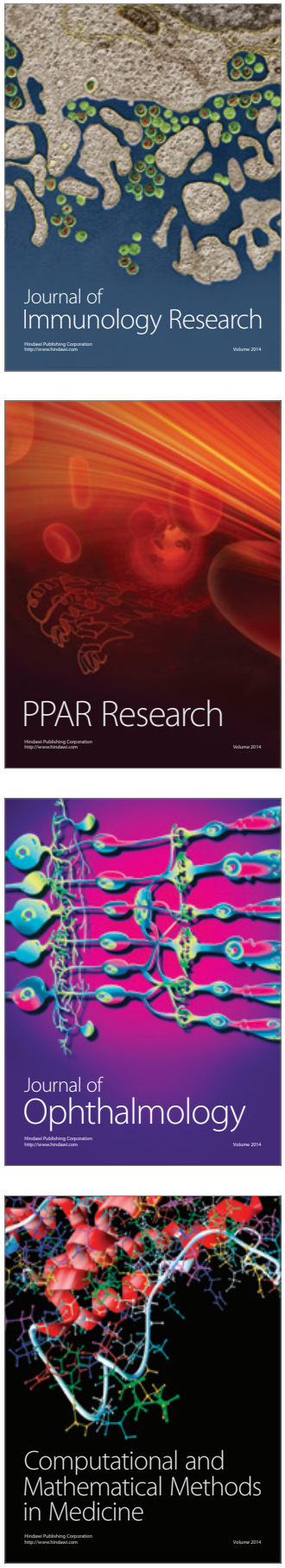

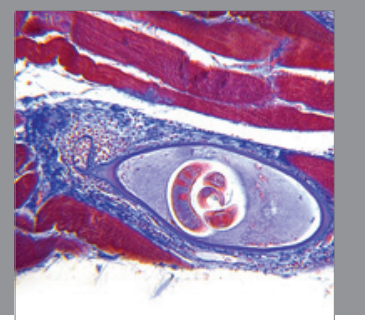

Gastroenterology

Research and Practice
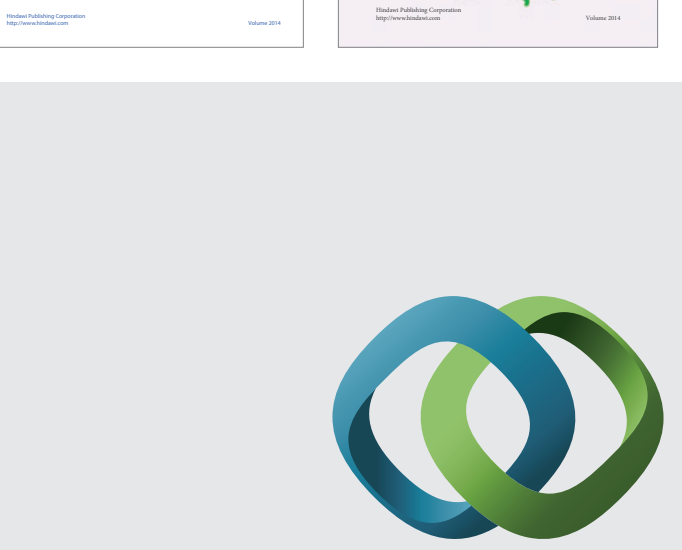

\section{Hindawi}

Submit your manuscripts at

http://www.hindawi.com
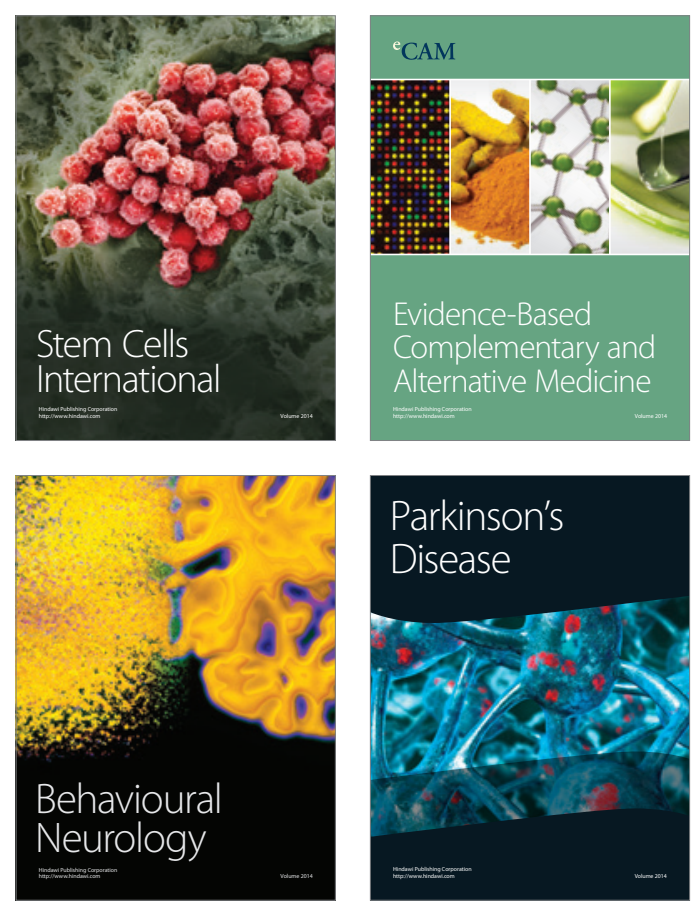

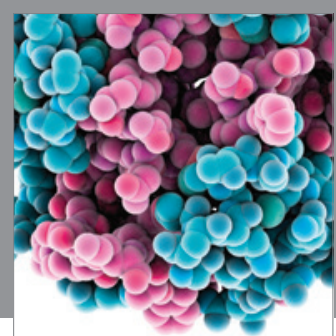

Journal of
Diabetes Research

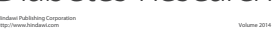

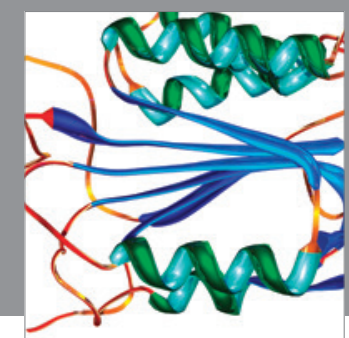

Disease Markers
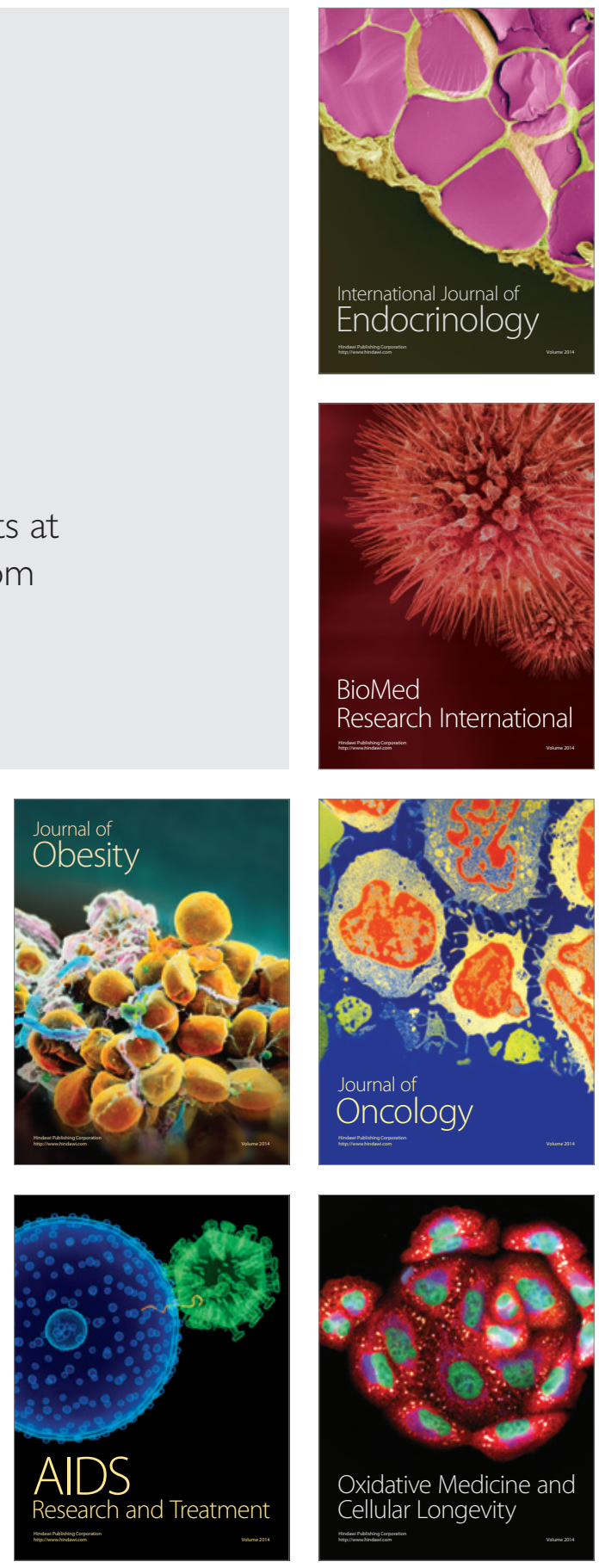